\title{
Motivación y el desempeño de los docentes del instituto de educación superior tecnológico publico Chocope-2021
}

\author{
Ada Luz Clotilde Ardiles Abanto \\ aardiles@ucv.edu.pe \\ https://orcid.org/0000-0002-6432-6291 \\ Universidad César Vallejo \\ María Elena Alva Loyola \\ malva@ucv.edu.pe \\ https://orcid.org/0000-0002-3034-2170 \\ Universidad César Vallejo \\ Dulio Oseda Gago \\ doseda@ucv.edu.pe \\ https://orcid.org/0000-0002-3136-6094 \\ Universidad César Vallejo
}

\section{RESUMEN}

La investigación parte del objetivo determinar la relación que existe entre la motivación y el desempeño de los docentes del instituto de educación superior tecnológico publico Chocope, 2021. La investigación fue de enfoque cuantitativo, tipo básica, nivel correlacional y diseño descriptivo correlacional. La población de estudio estuvo conformada por 44 docentes del instituto de educación superior tecnológica público Chocope durante el periodo 2021 y la muestra fue censal. Como técnicas se trabajó con la encuesta y el instrumento cuestionarios, los cuales previa a su aplicación de confiabilizaron y validaron; de donde se desprende que existe una correlación altamente significativa entre la motivación y el desempeño de los docentes del instituto de educación superior tecnológico publico Chocope, 2021.

Palabras clave: motivación; intrínseca; extrínseca; desempeño de docentes. 


\title{
Motivation and performance of the teachers of the Chocope-2021 public technological higher education institute
}

\begin{abstract}
The research starts with the objective of determining the relationship between the motivation and the performance of the teachers of the public institute of higher technological education Chocope, 2021. The research was of a quantitative approach, basic type, correlational level and correlational descriptive design. The study population consisted of 44 teachers from the Chocope public technological higher education institute during the 2021 period and the sample was census. As techniques we worked with the survey and the questionnaires instrument, which prior to its application were made reliable and validated; From which it can be inferred that there is a significantly significant correlation between the motivation and performance of the teachers of the Chocope public technological higher education institute, 2021.
\end{abstract}

Keywords: Motivation, intrinsic, extrinsic, teacher performance.

Artículo recibido: 30 noviembre. 2021 Aceptado para publicación: 29 diciembre 2021

Correspondencia: aardiles@ucv.edu.pe Conflictos de Interés: Ninguna que declarar 


\section{INTRODUCCION}

Existe una nueva realidad en la docencia, derivada de la crisis de la pandemia covid-19, que ha dado origen a una nueva forma de enseñanza. Este cambio radical e imprevisto ha obligado a las diferentes instituciones a utilizar todas las herramientas disponibles para adaptarse, no solo en infraestructura sino también en el factor más preciado, el factor humano.

Este último ha cobrado mayor relevancia en este nuevo contexto, dado que, al ser la base, estructura y motor de desarrollo de cualquier institución, ha impulsado, logrado y consolidado metas, que, en un primer momento, parecían muy difíciles de alcanzar. Es imperativo entender que los docentes son los principales protagonistas de esta magnánima tarea, y quien se afirma, factor fundamental para el desarrollo de la misma, entendiéndose así, como el factor humano, que da la razón de ser de cualquier institución.

Sin embargo, este factor, ha demostrado ser sensible como cualquier otro, pero con la particularidad, que bajo un correcto liderazgo genera resultados rápidos, concretos, eficientes y sobre todo cumple objetivos, que se traduce finalmente, en el desempeño. Nace así una necesidad de gestionar, promover, administrar, fomentar e impulsar este factor si se pretende lograr un mayor desempeño.

Esta nueva realidad ha demandado a las diferentes instituciones a implementar un plan de acción dirigido a la motivación, como instrumento que impulsa y fomenta el desempeño del cuerpo docente, preservando así la eficiencia y eficacia de tan valioso factor. Aseveramos entonces, que la motivación de los docentes cobra relevancia y es una de las principales preocupaciones, y debería ser, de quienes gestionan y administran una institución.

Entendemos entonces, que un cuerpo docente oportunamente motivado, puede generar un aumento significativo de los niveles de su desempeño, sin alterar la calidad de su trabajo. Esta acción se traduce finalmente, en el desempeño mismo de la institución. La actual pandemia del covid-19 evidencio que un cuerpo docente bajo un correcto liderazgo, con soporte idóneo y herramientas oportunas, generan un ahorro de recursos, cumple objetivos y brinda una educación de calidad. Sin embargo, el desarrollo de estas características requiere indicadores, planes de gestión y herramientas acorde a la dinámica institucional, para identificar así, los diferentes factores internos y externos, que pueden influir sobre esta. 
Por eso es necesario el desarrollando de estrategias y planes de acción para el grupo humano en base a datos concretos y objetivos medibles. Según Robbins (1999) define la motivación como la voluntad o la intensión de generar niveles altos de esfuerzo direccionados a las metas de la organización a quien pertenece, generado por la necesidad de saciar o cubrir algún deseo o anhelo. Herrera, Ramírez, Roa y Herrera (2004), definen la motivación como la representación de un hecho o conducta que señala o induce a un individuo a que inicie una acción concreta (activación), dirigido a un objetivo (dirección) y que este sea persistente hasta alcanzarlo (mantenimiento).

Bohorquez (2020) expone su relevancia al manifestar que el capital humano se considera el activo de más alto valor y significancia de la empresa o institución, dado que un trabajador complacido y motivado, mantiene o mejora la productividad, debido a la predisposición de su comportamiento con el compromiso que tiene con la empresa. En base a lo anterior se plantea la siguiente pregunta de investigación, el problema general: ¿Qué relación existe entre la motivación y el desempeño de los docentes del instituto de educación superior tecnológico publico Chocope, 2021 ?

El Objetivo general de la investigación consistió en determinar la relación que existe entre la motivación y el desempeño de los docentes del instituto de educación superior tecnológico publico Chocope, 2021. Y la hipótesis general de la investigación existe una relación positiva y significativa entre la motivación y el desempeño de los docentes del instituto de educación superior tecnológico publico Chocope, 2021.

Respecto a las bases teórico científicas, se consultaron diferentes fuentes bibliográficas relevantes para desarrollar el marco conceptual correspondiente. Así tenemos, para la variable motivación definida por Sum (2015), quien la define como el conjunto de impulsos que posee una persona que son originados por los diferentes estímulos que pueden venir por factores externos o internos, que inciden de diferentes maneras en la forma de como un individuo es motivado, que varía de persona a persona. Se expone entonces que esta dependencia de las necesidades que se obtengan, así como sus preferencias, creencias, valores, entre otras características, influyen en la satisfacción laboral. Este proceso inicia en identificando las necesidades de una persona, las incomodidades y problemáticas que presenta y su comportamiento.

Según Robbins \& Coulter (2010), definen a la motivación como el proceso a través el cual el nivel esfuerzo que tiene un individuo estimulados, direccionados y mantenidos 
hacia el desarrollo de una meta concreta.

Para Heredia (2004) la definición de la motivación implica a que es un conjunto de factores que generan comportamientos de origen fisiológico, psicológico, social y cultural. Determinando así que la motivación en cada individuo es diferente, acorde a las necesidades y funciones de una persona a otra, pero que finalmente producen diversos tipos de comportamiento. Es decir, la capacidad de cada individuo para alcanzar objetivos y metas es diferente. Así mismo, la motivación es variable en cada individuo acorde a diferentes necesidades que a su vez originan una serie de comportamientos (Linares 2017).

Montes de Oca (2011) la define como que el conjunto de acciones y procedimientos debidamente programados, de carácter regulativo y con dirección acorde a condiciones específicas debidamente desarrolladas a nivel de educación, aprendizaje y enseñanza que contiene. Se define, así como la finalidad de fomentar un sentimiento en un grupo de individuos. Así mismo, la motivación se define como el impulso que empuja a los individuos a desarrollar diferentes tareas. La importancia de la motivación radica en ser un potente agente que impulsa o mejorara la efectividad del capital humano, en consecuencia, es una herramienta intangible que produce un aumento de la productividad esperada. El desarrollo o implementación se da a través de un conjunto de acciones orientadas al factor humano que trae en su implementación un aumento de los principales indicadores cuantitativos y cualitativos.

Para David y Newstrom (2008), dimensionan a la variable motivación acorde al estímulo laboral, incentivo, contribuciones, recompensas, castigos, liderazgo, responsabilidades y oportunidades al desarrollo. Así tenemos para la dimensión estimulo laboral como el conjunto de estímulos positivos o negativos, que ayudan a reforzar, inhibir o eliminar un comportamiento concreto. Para el incentivo como dimensión se le asigna a la acción de paga acorde a tareas asignadas por la institución de los trabajadores, entendiéndose así como nóminas, premios, beneficios sociales, estabilidad en el cargo o elogios. La contribución en su forma de dimensión, se le entiende como el conjunto de pagos que acciona cada trabajador con fin a la institución que tiene un valor de beneficio que varía. La recompensa, se entiende en un paquete de beneficios que la organización pone en práctica a sus integrantes. La dimensión definida como castigo, se le asigna a un conjunto de medidas disciplinarias que obedecen a la conducta de las personas. Liderazgo se 
entiende como el conjunto de acciones en el cual una persona influye en las actividades en un grupo de individuos para dirigir y coordinar esfuerzos hacia el logro de metas. La dimensión responsabilidad responde a una acción que contiene sabiduría y habilidad ante aquello que solamente a uno le compete, es decir que lo puede delegar mas solo compartir. Finalmente, la dimensión denominada oportunidad al desarrollo, a todo suceso en la cual existe la oportunidad de lograr algún tipo de mejora en el marco laboral. En este sentido podemos evidenciar que las dimensiones de la motivación son aquellos factores que inciden en relación directa en el comportamiento del individuo y como estos interactúan con sus semejantes. Para el análisis de la motivación del presente trabajo de investigación, se considera el análisis de las dimensiones de estímulo laboral, contribuciones, liderazgo, responsabilidades y oportunidades de desarrollo. Así mismo, para desarrollar la variable desempeño se consultaron diferentes fuentes bibliográficas relevantes para desarrollar el marco conceptual correspondiente. Así tenemos, para esta variable se define como el cumplimiento de los objetivos planeados acorde a la mínima cantidad de recursos necesarios utilizados, sin alterar la calidad de la misma, en un determinado tiempo.

Según Robbins y Judge (2013), la define como al nivel más alto de la conducta de una institución, debido a que un trabajador o colaborador se considera productivo si cumple sus objetivos o tareas asignadas. Considerándose así, al desempeño como la convergencia de eficiencia y eficacia de una actividad de acción recurrente o de un objetivo específico. Para Montalvo (2011), el desempeño del docente es la calificación que este obtiene en calidad de profesional acorde a la medición de diferentes y variadas cualidades tanto en conductas en el trabajo, puntualidad, comportamiento, disposición para el trabajo, disciplina, compromiso institucional, desarrollo pedagógico, innovación entre otras. Se define así al desempeño como el accionar de cumplir un deber asignado, este accionar es propio a la profesión, oficio o cargo asignado dentro de la institución, y pugna a actuar, laborar en la actividad que se realiza satisfactoriamente. Existen diferentes definiciones, no obstante, el término de desempeño se viene acuñando en forma concreta a la calidad total. Hoy el término desempeño, no solo constituye un ratio laboral sino también un instrumento para medir los niveles de efectividad.

Dentro del campo gerencial, estos direccionan la orientación que deben seguir los niveles de desempeño según el capital humano. Esta característica no solo se limita para la elección de puestos de trabajos, sino también para garantizar el mejor servicio que se 
genera. En el contexto pedagógico, el desempeño docente es la actividad relevante que este realiza acorde al producto de la interacción con sus estudiantes en el aula de clase, utilizando una serie de metodologías y estrategias, para lograr el éxito en sus propuestas curriculares de aprendizaje. Acorde a la importancia que esta genera, Javier et al. (2019) manifiesta en sus implicancias que genera para las instituciones, al desarrollar un sistema de competencias relevantes al desarrollo en los trabajadores, a la vez que ellos crecen en lo personal y profesional. Así tenemos que para la presente investigación, las dimensiones de la variable del desempeño del docente, toma los criterios e indicadores del sistema de evaluación de desempeño docente del Ministerio de Educación del Perú (2007) donde el ente referido, toma en consideración tres dimensiones.

El primero se refiere a la planificación del trabajo que se denomina al conjunto de acciones planificadas dentro del proceso de enseñanza acorde al aprendizaje, donde se relaciona los conocimientos que el docente imparte, acorde a las capacidades pedagógicas para que se concrete un correcto aprendizaje del alumnado en óptimas condiciones y sin alterar la calidad de este proceso. También podemos definirla como la preparación de la enseñanza acorde a las planificaciones hechas y el efecto que esta genera durante el desarrollo del proceso de enseñanza y de aprendizaje en el aula. El segundo es la gestión de los procesos de enseñanza y aprendizaje que de acuerdo al Ministerio de Educación del Perú (2007) que comprende al conjunto de factores que interactúan acorde a la acción de aprender y al clima o ambiente físico o abstracto donde interactúa el docente con su alumnado Asimismo, se refiere las diferentes capacidades que tiene el docente para ejercer la acción de pedagogía y que esta se acrecienta durante las sesiones de aprendizaje, mediante la disciplina, comunicación clara y sencilla de los contenidos con la aplicación de estrategias y metodologías que colocan al estudiante en el centro del proceso de enseñanza- aprendizaje; aquí se da importancia al uso del tiempo y del espacio, así como a la evaluación y meta evaluación o meta cognición. El tercero son las responsabilidades profesionales que Implica "el cumplimiento de responsabilidades laborales y profesionales previamente definidas. Estas corresponden al compromiso del docente con los procesos de aprendizaje, el afán de superación profesional, la capacidad para reflexionar sobre su práctica y la de sus colegas y su identificación con las metas y desempeño institucional. Asimismo, comprende el apoyo y comunicación con los estudiantes, padres de familia y comunidad (MINEDU, 2007); las responsabilidades 
profesionales implican la reflexión sobre la práctica docente en el aula, así como las relaciones profesionales que el docente desarrolle con sus colegas, manejando información actualizada sobre su profesión y como éste propicie la colaboración y respeto con los padres, además de asumir dichas responsabilidades en la orientación de sus educandos.

\section{METODOLOGIA}

La investigación es de tipo básica dado que su propósito es la de generar un conocimiento nuevo sobre un hecho abstraído de la realidad según lo asevera Bunge (1971). Así mismo, el enfoque considerado para esta investigación es el cuantitativo, dado que utiliza la recolección de datos con medición numérica basándose en técnicas estadísticas para interpretar la relación entre un entre la motivación y el desempeño de los docentes del instituto de educación superior tecnológico publico Chocope, 2021.

El método es el hipotético-deductivo dado que observa el fenómeno propuesto, establece una hipótesis y se deduce las consecuencias de la misma.

El diseño empleado es el no experimental, dado que en la investigación no experimental resulta imposible manipular variables, como señala Kerlinger (1981). Por tanto, esta investigación solamente se observará fenómenos tal como se dan en su contexto natural para posteriormente ser analizados. Se ha utilizado el diseño descriptivo - correlacional.

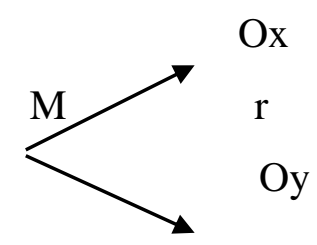

$\mathrm{M}=$ Muestra

Ox: Motivación (Variable independiente)

Oy: Desempeño (Variable dependiente)

r: relación que existe entre variables

La población de estudio está conformada por 44 docentes del instituto de educación superior tecnológica público Chocope durante el periodo 2021.

La presente investigación recabo información personal e institucional, donde se evaluó a 44 docentes del instituto de educación superior tecnológica público Chocope que cumplieron con el criterio de inclusión y exclusión. 
Unidad de análisis: Docentes del instituto de educación superior tecnológica público Chocope.

El presente trabajo se desarrolló con el total de la población de 44 docentes seleccionados acorde al criterio de muestreo no probabilístico por conveniencia, con la participación de los docentes que proporcionaron la información necesaria para el desarrollo de la presente investigación.

La técnica de recopilación de información usada es la encuesta y mediante un cuestionario se procedió a encuestar a diferentes docentes del instituto de educación superior tecnológica público Chocope. El cuestionario tendrá diversas preguntas con el fin de recopilar información esencial para saber la relación que existe entre la motivación y el desempeño laboral.

Se realizó una encuesta con un cuestionario de 20 ítems a 44 docentes del instituto de educación superior tecnológica público Chocope, donde se calculó el coeficiente de alfa de Cronbach del instrumento, para medir la fiabilidad del test, ya que fue elaborada en escala tipo Likert con cinco alternativas de respuesta.

En una primera instancia se evaluó la confiabilidad de los instrumentos mediante una muestra piloto de 15 docentes. Información que se procesó mediante el programa IBM SPSS v.25, estimando un valor de 0.930 para la variable motivación y 0.914 para la variable desempeño representando un nivel de confiabilidad aceptable para los instrumentos evaluados. Es así que, al obtener un resultado positivo de la muestra piloto, se procedió a aplicar el instrumento mediante la técnica de la encuesta a toda nuestra población en estudio.

La primera parte del cuestionario, mide la variable motivación. Esta se compone de 10 preguntas, las cuales corresponden a sus 4 dimensiones. Así mismo, la segunda parte del cuestionario, mide la variable desempeño del docente, que también cuenta con 10 preguntas, las cuales corresponden a sus 4 dimensiones.

De un total de 44 casos estudiados, se evidencia que el coeficiente Alfa de Cronbach que se obtiene es de 0,961, lo cual permite evidenciar que el cuestionario que mide las variables motivación y desempeño posee muy alta fiabilidad.

El análisis de ambos instrumentos en forma individual y en conjunto del total de sus ítems, se observa que posee un alto nivel de confiabilidad, por lo que es idóneo para su aplicación a toda la muestra de la presente investigación. 


\section{RESULTADOS Y DISCUSIÓN}

Distribución de los docentes del instituto de educación superior tecnológico publico

Chocope según niveles alcanzados en la variable motivación

Tabla 1. Niveles alcanzados de la variable motivación

\begin{tabular}{|l|c|c|}
\hline \multicolumn{1}{|c|}{ Nivel } & Frecuencia & Porcentaje \\
\hline Muy en desacuerdo & 4 & $9,1 \%$ \\
\hline En desacuerdo & 0 & $0,0 \%$ \\
\hline Indeciso & 9 & $20,5 \%$ \\
\hline De acuerdo & 24 & $54,5 \%$ \\
\hline Muy de acuerdo & 7 & $15,9 \%$ \\
\hline Total & $\mathbf{4 4}$ & $\mathbf{1 0 0 , 0 \%}$ \\
\hline
\end{tabular}

Fuente: Encuesta aplicada. Elaboración: El autor

En la tabla 1 podemos evidenciar la distribución de los docentes del instituto de educación superior tecnológico publico Chocope según niveles alcanzados en la variable motivación acorde a los puntajes obtenidos. Se ubican los niveles muy en desacuerdo, en desacuerdo, indeciso, de acuerdo y muy de acuerdo. Así tenemos que el 20,5\% (9) de los docentes se sienten indecisos a nivel motivacional, mientras que 54,5\% (24) están de acuerdo en estar motivados; estos resultados indican que un docente motivado genera una experiencia gratificante su desenvolvimiento en la institución. Sin embargo, solo el 9\% (4) están muy en desacuerdo en el nivel de motivación, estos docentes encuentran una mala experiencia o ausencia de esta en la institución. No se precisa docentes en desacuerdo.

La prueba de normalidad, es un instrumento estadístico que permite determinar la idoneidad del instrumento a utilizar para la comprobación de hipótesis. Se muestran las pruebas de normalidad para las variables motivación y desempeño laboral.

Tabla 2. Prueba de normalidad para la variable motivación

\begin{tabular}{|l|c|c|c|c|c|c|}
\hline \multicolumn{1}{|l|}{ Pruebas de normalidad } \\
\hline & Estadístico & $\mathrm{gl}$ & Sig. & Estadístico & $\mathrm{gl}$ & Sig. \\
\cline { 2 - 7 } &, 192 & 44 &, 000 &, 824 & 44 &, 000 \\
\hline Motivación &, 199 & 44 &, 000 &, 831 & 44 &, 000 \\
\hline Estimulo laboral &, 300 & 44 &, 000 &, 783 & 44 &, 000 \\
\hline Contribución &, 258 & 44 &, 000 &, 888 & 44 &, 000 \\
\hline Liderazgo &, 206 & 44 &, 000 &, 913 & 44 &, 003 \\
\hline $\begin{array}{l}\text { Oportunidad al } \\
\text { desarrollo }\end{array}$ & & & & & \\
\hline \begin{tabular}{l} 
a. Corrección de significación de Lilliefors \\
\hline
\end{tabular}
\end{tabular}

Fuente: Encuesta aplicada. Elaboración: El autor 
Se evidencia en la tabla 2 , los resultados de la prueba de normalidad de la variable motivación. Dado que el número de datos es mayor a 35, se ha utilizado la prueba de normalidad de Kolmogorov-Smirnov, donde los resultados exponen un p. valor (0.000), que es menor al alpha (0.05), lo cual indica que los datos de la variable motivación no siguen una distribución normal y se utilizará Estadística No Paramétricas. Es decir, para esta variable se va utilizar Rho-Spearman.

Análisis descriptivo para la variable desempeño laboral

Distribución de los docentes del instituto de educación superior tecnológico publico Chocope según niveles alcanzados en la variable desempeño laboral

Tabla 3. Niveles alcanzados de la variable desempeño laboral

\begin{tabular}{|l|c|c|}
\hline \multicolumn{1}{|c|}{ Nivel } & Frecuencia & Porcentaje \\
\hline Muy en desacuerdo & 4 & $9,1 \%$ \\
\hline En desacuerdo & 1 & $2,3 \%$ \\
\hline Indeciso & 2 & $4,5 \%$ \\
\hline De acuerdo & 31 & $70,5 \%$ \\
\hline Muy de acuerdo & 6 & $13,6 \%$ \\
\hline \multicolumn{1}{|c|}{ Total } & $\mathbf{4 4}$ & $\mathbf{1 0 0 , 0 \%}$ \\
\hline
\end{tabular}

Fuente: Encuesta aplicada. Elaboración: El autor

En la tabla 3 podemos evidenciar la distribución de los docentes del instituto de educación superior tecnológico publico Chocope según niveles alcanzados en la variable desempeño laboral acorde a los puntajes obtenidos. Se ubican los niveles muy en desacuerdo, en desacuerdo, indeciso, de acuerdo y muy de acuerdo. Así tenemos que el 4,5\% (2) de los docentes se sienten indecisos a nivel de su desempeño laboral, mientras que 70,5 \% (31) están de acuerdo en el nivel de desempeño laboral existente; estos resultados indican que un docente con un alto desempeño genera una experiencia eficiente en las tareas asignadas en la institución. Sin embargo, solo el 9\% (4) están muy en desacuerdo en el nivel de desempeño laboral y solo el $2 \%$ (1) de docentes están en desacuerdo del nivel del mismo.

Tabla 4. Prueba de normalidad para la variable desempeño laboral

\begin{tabular}{|l|c|c|c|c|c|c|}
\hline \multicolumn{7}{|c|}{ Pruebas de normalidad } \\
\hline & \multicolumn{3}{|c|}{ Kolmogorov-Smirnov ${ }^{\text {a }}$} & \multicolumn{3}{c|}{ Shapiro-Wilk } \\
\cline { 2 - 7 } & Estadístico & gl & Sig. & Estadístico & gl & Sig. \\
\hline Desempeño laboral &, 303 & 44 &, 000 &, 738 & 44 &, 000 \\
\hline a. Corrección de significación de Lilliefors
\end{tabular}

Fuente: Encuesta aplicada. Elaboración: El autor 
Se evidencia en la tabla 4, los resultados de la prueba de normalidad de variable desempeño laboral. Dado que el número de datos es mayor a 35, se ha utilizado la prueba de normalidad de Kolmogorov-Smirnov, donde los resultados exponen un p. valor (0.000), que es menor al alpha (0.05), lo cual indica que los datos de la variable desempeño laboral no siguen una distribución normal y se utilizará Estadística No Paramétricas. Es decir, para esta variable se va utilizar Rho-Spearman.

Prueba de hipótesis. Para realizar la correlación entre las variables motivación y la productividad laboral se tomaron en cuenta la prueba de normalidad de KolmogorovSmirnov, donde evidencio que los datos de la variable motivación y la variable desempeño laboral no siguen una distribución normal. Por tanto, se utilizará el coeficiente Rho de Spearman para determinar el nivel de relación existente entre las variables motivación y desempeño laboral de los docentes del instituto de educación superior tecnológico publico Chocope, 2021. El análisis estadístico de los resultados se presenta en la siguiente tabla:

Tabla 5. Correlación entre las variables motivación y la productividad laboral

\begin{tabular}{|c|c|c|c|c|}
\hline \multicolumn{3}{|c|}{ Rho de Spearman } & Motivación & Desempeño laboral \\
\hline \multirow{6}{*}{$\begin{array}{c}\text { Rho de } \\
\text { Spearman }\end{array}$} & \multirow{3}{*}{ Motivación } & $\begin{array}{l}\text { Coeficiente de } \\
\text { correlación }\end{array}$ & 1,000 &, $630^{* *}$ \\
\hline & & Sig. (bilateral) & . & ,000 \\
\hline & & $\mathrm{N}$ & 44 & 44 \\
\hline & \multirow{3}{*}{$\begin{array}{c}\text { Desempeño } \\
\text { laboral }\end{array}$} & $\begin{array}{l}\text { Coeficiente de } \\
\text { correlación }\end{array}$ &, $630^{* *}$ & 1,000 \\
\hline & & Sig. (bilateral) & ,000 & 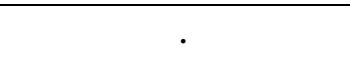 \\
\hline & & $\mathrm{N}$ & 44 & 44 \\
\hline
\end{tabular}

Fuente: Encuesta aplicada. Elaboración: El autor

En la tabla 5 se identifica una relación directa y significativa entre el nivel de motivación y desempeño laboral de los de los docentes del instituto de educación superior tecnológico publico Chocope, 2021. Los resultados evidencian un nivel de correlación moderada ( $\mathrm{r}=$ 0,630). La existencia de correlación también se puede apreciar en el análisis de dispersión mostrado en la siguiente figura, en la cual se observa que existe aglomeración o tendencia de los puntajes, hacia arriba a la derecha característica de una correlación positiva 


\section{CONCLUSIONES}

1) Se ha determinado que existe una relación positiva fuerte y significativa entre el estímulo laboral y el desempeño de los docentes del instituto de educación superior tecnológico publico Chocope, 2021.

2) Existe una relación positiva fuerte y significativa entre la contribución y el desempeño de los docentes del instituto de educación superior tecnológico publico Chocope, 2021.

3) Existe una relación positiva muy fuerte y significativa entre el liderazgo y el desempeño de los docentes del instituto de educación superior tecnológico publico Chocope, 2021.

4) Existe una relación positiva fuerte y significativa entre la oportunidad al desarrollo y el desempeño de los docentes del instituto de educación superior tecnológico publico Chocope, 2021.

\section{REFERENCIAS BIBLIOGRAFICAS}

Anticona, M. y Gaitán, L. (2016). “Aplicación de un modelo de gestión por competencias para mejorar el desempeño laboral de los trabajadores del grupo educativo Las Américas S.R. L de la ciudad de Trujillo -2016. Trujillo.

Bohorquez, E., Perez, M., Caiche, W., \& Rodriguez, A. (2020) La motivación y el desempeño laboral: El capital humano como factor clave en una organización. Universidad y $\quad$ Sociedad, 12(3), $385 \quad-\quad 390$. http://scielo.sld.cu/scielo.php?script=sci_arttext\&pid=S2218$36202020000300385 \& \operatorname{lng}=\mathrm{es} \& \mathrm{nrm}=\mathrm{iso}$

Choque, M. (2018) Plan estratégico de tecnologías de la información orientado al éxito de proyectos en instituciones de educación superior. Yachay - Revista Científico Cultural, 7(01), 424-434. https://doi.org/10.36881/yachay.v7i01.95

Heredia, A. (2004). Administración de recursos humanos para alto desempeño. México: trillas

Linares, J. (2017) Motivación laboral y desempeño laboral en el centro de salud la Huayrona 2017. [Tesis de maestría, Universidad Cesar Vallejo], Repositorio institucional de la Universidad Cesar Vallejo. https://repositorio.ucv.edu.pe/bitstream/handle/20.500.12692/9005/Linares_MJS .pdf? sequence $=1$ 
Robbins, P y Coulter, M. (2010). Administración. (2 ${ }^{\mathrm{a}}$ ed.). México: Editorial Prentice Hall.

Marco del buen desempeño del docente, un buen maestro cambia la vida (2007). Minedu.

Montalvo, W. (2011). El clima organizacional y su influencia en el desempeño docente en · las Instituciones Educativas del nivel de educación secundaria de la UGEL 15 de Huarochirí - 2008. Lima: Universidad Enrique Guzmán y Valle.

Meza, E. (2018) Clima organizacional y desempeño laboral en empleados de la universidad Linda Vista, en Chiapas [Tesis de maestría, Universidad de Montemorelos]. Repositorio institucional de la Universidad de Montemorelos, México.

http://dspace.biblioteca.um.edu.mx/xmlui/bitstream/handle/20.500.11972/901/T esis\%20Eloina\%20Meza\%20Cruz.pdf?sequence=1\&isAllowed=y

Ramírez, O., Patiño, J., Patiño, M., \& Cuellar, M. (2020) Medición del comportamiento laboral y su impacto en la productividad. Computación y Sistemas, 24(3), 1305 1312.

http//doi.org/10.13053/CYS-24-3-3489

Rivera, L. (2021) Gestión del conocimiento y pedagógico en la calidad educativa de una RED Educativa de la Región Callao. [Tesis de doctorado, Universidad Cesar Vallejo]. Repositorio institucional de la Universidad Cesar Vallejo. https://repositorio.ucv.edu.pe/bitstream/handle/20.500.12692/65131/Rivera_ML P-SD.pdf?sequence $=1 \&$ isAllowed $=\mathrm{y}$

Sum, I. (2015). Motivación y Desempeño Laboral. [Tesis de pregrado, Universidad Rafael Landívar. Quetzaltenango]. Universidad Rafael Landívar. Quetzaltenango. http://recursosbiblio.url.edu.gt/tesisjcem/2015/05/43/SumMonica.pdf 